\title{
Preparation of super hydrophobic coating for insulator against icing flashover
}

Tao Yao

\section{School of Energy Power and Mechanical Engineering, North China Electric Power University, Baoding, 071000, China 380085362@qq.com}

\begin{abstract}
: the insulator is a kind of important electric components, because it plays a role in electrical insulation and plays a supporting role in mechanical, power system and even the development of the whole society to a higher degree of dependence on the performance of insulators, the requirements are also rising. However, in recent years, China's domestic air pollution is serious, the weather conditions are complex, the icing flashover phenomenon has become a serious performance of insulator safety accidents. However, the proposed super hydrophobic materials in this paper, not only the anti icing flashover performance is better than the commonly used coating, and preparation process is sample, high performance, and low cost, which is expected to become a substitute for insulator coating now.

the super hydrophobic coating is considered to be one of the important methods to solve the problem of flashover electrical equipment, so the study of high hydrophobicity flashover, super hydrophobic coating with high performance, simple process, low cost plays an important role in electrical equipment and power industry. In this paper, we will use a kind of method which is more powerful, graphene was prepared by electrochemical stripping and poly-two methyl siloxane in a certain proportion to prepare superhydrophobic surface. In this paper, the preparation of superhydrophobic surface, not only has excellent self-cleaning performance, but also has a very low corrosion current density. It is surprising that the superhydrophobic surface prepared by this method has good mechanical stability, and its surface can maintain the super hydrophobicity after the strong impact of the outside world. The method mentioned in this paper is not only capable of preparing a set of super hydrophobic surface self cleaning,mechanical stability is stable as a whole, it also provides a new idea for anti icing flashover of electrical equipment.
\end{abstract}

Key words: Superhydrophobic Coating; Graphene; Insulator; Corrosion; Resistance

\section{Introduction}

With the development of the electric power industry and the gradual improvement of the voltage level, the electrical equipment is playing a more and more important role in people's daily production and life. Of which the insulator is an important part of power system, however, the phenomenon of icing flashover has a great influence on the mechanical and electrical properties of insulators, which will threaten the stability and security of power system operation, resulting in huge economic losses. Take snow disaster in South China in 2008 as an example, the ice coating accidents spread to 13 provinces, State Grid economic losses in the accident up to 10 billion 450 million Yuan, and in daily life, line trip, outage accidents also occur caused by icing ${ }^{[1]}$, it causes a great impact on people's life. Therefore, it has become one of the most urgent problems to solve the problem of icing flashover. However, there are still a lot of defects in the deicing and anti icing methods currently used, and the efficiency is low and the energy consumption is large.

Super hydrophobic coating is a kind of water elapse, reduce the drop freezing probability, thereby delaying the formation of ice coating, especially in the early stage of icing, it has great effect on quality and morphology ${ }^{[2]}$, so the development of super hydrophobic coating has a strong advantage in the anti icing of insulators. The preparation method of this study not only has high hydrophobic performance, preparation process is simple, low cost, but also has prominent effect on corrosion resistance and impact resistance.

Super hydrophobic surface refers to the water contact angle greater than 150 degrees, the rolling angle less than 10 degrees of surface. Because of the super hydrophobic surface in the super hydrophobic surface, refers to the water contact angle greater than 150 degrees, the rolling angle of less than 10 degrees of surface. Because of 
its many applications such as cleaning ${ }^{[3][4]}$, corrosion resistant ${ }^{[5][6]}$, anti icing ${ }^{[7]}$, oil-water separation ${ }^{[8]}$ and so on, more and more attention has been paid to it. Many studies have revealed that the super hydrophobic surface is produced by proper surface roughness and low surface energy ${ }^{[9]}$, for example, a large number of artificial superhydrophobic surfaces can be prepared by the method of layer by layer deposition ${ }^{[10]}$, on the basis of template ${ }^{[11]}$, Photolithography ${ }^{[12]}$, sol-gel method ${ }^{[13]}$. However, these artificial surfaces will lose their self-cleaning performance when subjected to the impact of water and dust particles ${ }^{[14]}$. In view of this phenomenon, it was proposed, for example, perfluorinated polymers such as perfluoro octanoic acid and perfluoro three chloro silane can be conveniently processed and applied to superhydrophobic surfaces,but also can be used to improve the performance of not resistance, but recent research shows that the long chain perfluorinated compoundshas the capacity of biological accumulation, it exists adverse effects for human ${ }^{[15]}$. Therefore, the preparation of non-toxic and low-cost non-fluorinated superhydrophobic materials is more popular with researchers.

Graphene is a single atom thick film composed of SP2 hybridized carbon atoms, which has many unique physical and chemical qualities ${ }^{[16]}$, for example, high thermal conductivity, good thermal / chemical stability and excellent mechanical modulus. Surprisingly, the results show that the graphene material has a wonderful hydrophobic ${ }^{[17]}$. Many research groups have devoted themselves to the preparation of graphene superhydrophobic surfaces. For example: Rafiee and others obtain thermally reduced graphene oxide with tunable wettability by adjusting the solvent; Dong and others make super hydrophobic graphene film by using patterned Si substrates and microwave plasma $\mathrm{CVD}^{[19]}$; Nguyen and others repare graphene by ultrasonic stripping exfoliated graphite, and then obtain the super hydrophobic graphene sponge by dip coating method ${ }^{[20]}$; Wang and others prepare the

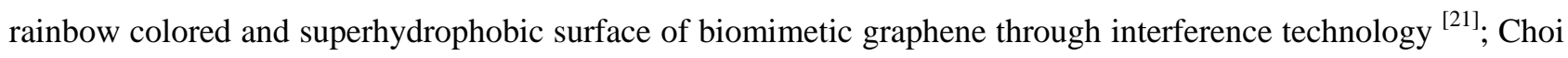
and others prepare superhydrophobic Aquastone graphene /Nafion hybrid membrane through conformational rearrangement ${ }^{[22]}$; Asthana anfd others produce Super hydrophobic coating by using Fluoropolymer dispersions of graphene / Carbon Black Composites ${ }^{[23]}$. However, it is not difficult to find that despite the increasing use of graphene to prepare superhydrophobic coatings, but the high cost and complexity of graphene has become an obstacle to the application of graphene superhydrophobic surface.

Recently, it is proposed that the electric chemical stripping of graphite is a low cost method for the preparation of grapheme ${ }^{[24]}$,this study has got more attention of research group. Parvez and others use electric chemical stripping graphene to produce a solution of the highly conducting electrode ${ }^{[25]}$. Wei and others demonstrate the potential applications of graphene in energy storage ${ }^{[26]}$. This paper proposes a simple and feasible, cost-effective way to form a super hydrophobic coating based on graphene with graphite as electrode, with (NH4) 2SO4 solution as electrolyte, get graphene from graphite by using electrochemical method, then, smear the mixture of graphene and poly (PDMS), the non fluorinated superhydrophobic surface can be realized. The superhydrophobic coatings prepared by this method can exhibit excellent self cleaning, and can continue to maintain the super hydrophobic properties after water impact and sand impact tests.

\section{Experimental steps}

\subsection{Material}

Poly (methyl siloxane) (PDMS) soluble in ethanol was obtained from China Hubei New World Co, Ltd. (two);

Ethanol (more than 99.7\%), 3- aminopropyltriethoxysilane (APTES) and $\mathrm{NaCl}$ China purchased from Shanghai Sinopharm Chemical Reagent Co. Ltd.

All chemicals are analytical grade reagents and are used at the time of receipt.

\subsection{Preparation of graphene}

In this paper, the method of preparing graphene is improved based on the existing preparation methods ${ }^{\text {[27] [28] }}$. In short, it is to use two graphite rods to form a dual electrode system routine, the electrolyte is $0.1 \mathrm{M}(\mathrm{NH} 4) 2 \mathrm{SO} 4$ 
solution, the distance between the electrode should maintain about $30 \mathrm{~mm}$, and then through the preparation of graphene by chemical stripping at a constant voltage of $10 \mathrm{~V}$, after the graphite peeling is completed, the obtained graphene product is dried up.

\subsection{Preparation of super hydrophobic composite coating}

The following specific preparation method of super hydrophobic coating: first, put $0.2 \mathrm{~g}$ polydimethylsiloxane into $10 \mathrm{~g}$ ethanol, and the solution is placed in ambient temperature mixing for 6 hours, and then adding $0.2 \mathrm{~g}$ graphene and $0.02 \mathrm{~g} 3$ - aminopropyltriethoxysilane by the same conditions, the same time stirring to form suspension the final solution. After completing the operation, the solution is coated on a substrate (Al6061 or stainless steel) with a disposable Pasteur pipette $(1 \mathrm{ml})$ and cured at 60 DEG C for 6 hours.

\subsection{Characterization experiment}

The morphology and structure of the samples were studied by scanning electron microscopy (Hitachi, S-3400N) and atomic force microscopy (Bruker, Multimode 8).

Measure contact angle and rolling angle, by depositing a thin layer of Au layer on the sample (about $5 \mathrm{~nm}$ ), optical contact angle measurement device for measuring water contact angle with SL200B- standard at ambient temperature (CAs) and rolling angle (SAs), drop $5 \mathrm{mu} \mathrm{L}$ drops in all the measurements by five different positions, measure the same samples and obtain the average CA value measurement;

\section{Results and discussion}

Graphene was prepared by electrochemical stripping of graphite. As shown in Figure 1a, graphite was removed in two graphite rods as anode and cathode. When the graphite rod is immersed in $0.1 \mathrm{M}(\mathrm{NH} 4)_{2} \mathrm{SO}_{4}$ solution, the $10 \mathrm{~V}$ voltage is applied, and a violent bubble is generated. As the $\mathrm{SO}_{4}{ }^{2-}$ and $\mathrm{H}_{2} \mathrm{O}$ tend to enter the graphite layer ${ }^{[27]}$, the anode graphite begins to dissociate into electrolyte. In this process, we believe that the reduction of $\mathrm{SO}_{4}{ }^{2-}$ anions and the autoxidation of $\mathrm{H}_{2} \mathrm{O}$ produce gaseous substances such as $\mathrm{SO}_{2}, \mathrm{O}_{2}$, etc., these gases can exert greater force in the graphite layer and separate the graphite layer with weak combination ${ }^{[28]}$, and ultimately form weak graphene bonding stripping graphite layer.

In order to study the quality of graphene, we performed atomic force microscopy and Raman spectroscopy. As shown in Figure 1b, the typical atomic force microscope image of an electrochemically exfoliated graphene sheet $(3.03 \mathrm{~nm})$ coated on a Si substrate. Figure 1C is a Raman spectrum of graphene powder, the peak of D $\left(1336 \mathrm{~cm}^{-1}\right)$ is caused by SP2 carbon atoms breathing patterns, and it may be activated because of problems such as structural disorder, functional or edge and other defects ${ }^{[28]}$, among them, the ID/IG ratio reflects the number of graphene defects. In this study, the ratio of ID/IG was 0.1, which was much smaller than that of chemical or thermally reduced graphene oxide $1.1-1.5^{[29]}$ and 0.4 of graphene in acidic solution using electrochemically exfoliated ${ }^{[25]}$. The results of Raman spectroscopy and atomic force microscopy show that the high quality graphene can be synthesized by electrochemical stripping method mentioned in this paper.
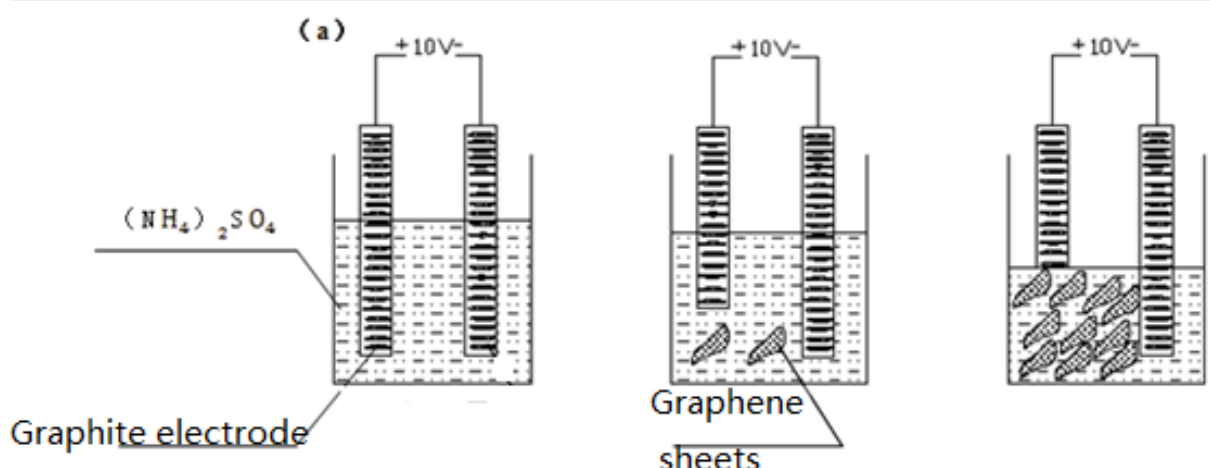

sheets 

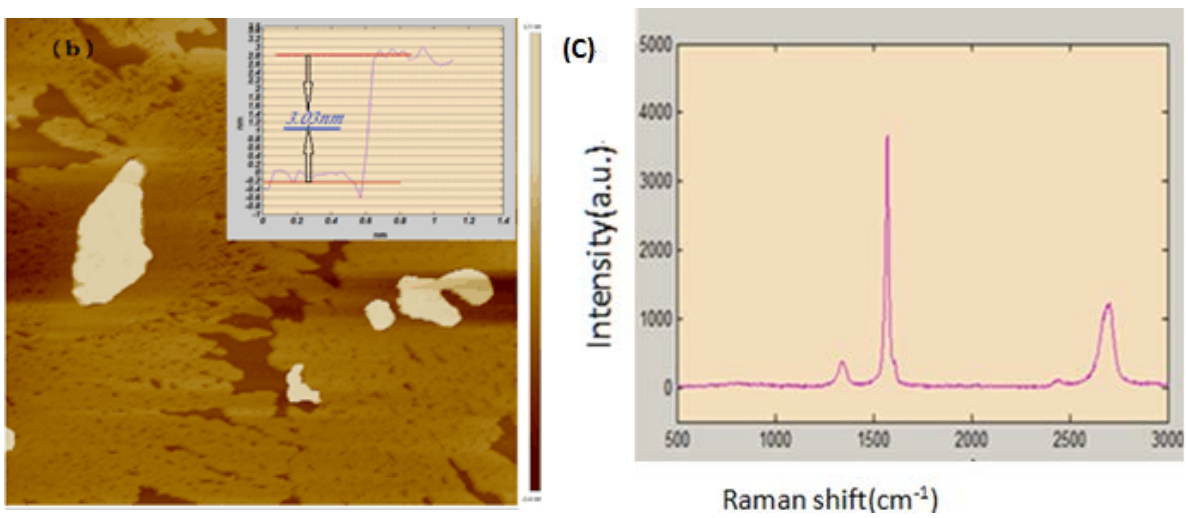

Fig. 1 (a) Schematic diagram of the mass production of graphene sheets through the electrochemical stripping of the two graphite electrodes $\quad$ (b) Microscope image of the atomic power of the graphene

\section{Raman spectroscopy of the graphene}

The suspension will be produced by adding the graphene into the ethanol solution containing PDMS and APTES. The addition of PDMS can not only provide low surface energy but also act as the adhesive to connect the graphene with each other and connect the graphene with the substrate. And APTES can improve the performance of PDMS. The superhydrophobic coating will be produced through the evaporation of ethanol and the solidification of PDMS for 6 hours in succession after covering the coating on the base material under the temperature of $60^{\circ} \mathrm{C}$. As shown in Fig. 2a, the water droplets (10 microliters) distributing randomly on the coating are nearly in the spherical shape, which means that the coating prepared is of good superhydrophobicity. It's necessary to measure the water contact angle and roll angle in order to implement the quantitative research of wetting condition: this kind of superhydrophobic coating shows a high contact angle of $160 \pm 2^{\circ}$ (Fig. $2 \mathrm{~b}$ ). At the same time, the water droplet will roll off the surface if the roll angle reaches $9^{\circ}$ (Fig. 2c), which means that the adhesion between the water droplet and the sample is low ${ }^{[30]}$. The high contact angle and low roll angle of the superhydrophobic coating are measured before the water droplet permeates into the surface prepared. As the Cassie-Baxter stated $^{[31]}$, the structure of the superhydrophobic coating shall be evaluated through SEM image. The nano-plate structure is shown in Fig. 2d.
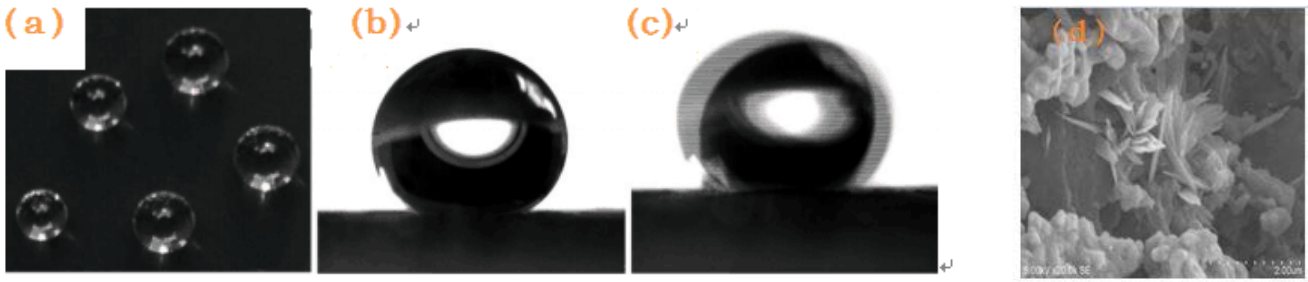

Fig. 2 (a) Picture of the water droplets deposited on the superhydrophobic surface (b) The contact angle is $160^{\circ}$

$\begin{array}{ll}\text { (c) The roll angle is } 9^{\circ} & \text { (d) SEM images on the superhydrophobic surface }\end{array}$

A large number of surfaces will get dirty easily due to the accumulation of the dust and dirt grains in the external environment, and the superhydrophobic coating is an efficient solution potentially in this case. This paper researches the self-cleaning property of the graphene superhydrophobic coating by taking sand as the polluted dust grains (as shown in Fig. 3). Firstly, spread a thin layer of sand on the superhydrophobic coating, and then place the water droplet on the contaminated surface gently. It's found in the experiment that the dust grains will be absorbed onto the surface of the water droplet immediately after they contact with the water droplet. The 
contaminant will be taken away by the water droplet with a clean surface left after the water droplet slides on the surface for several times, which proves that the graphene coating prepared is of excellent self-cleaning capability.

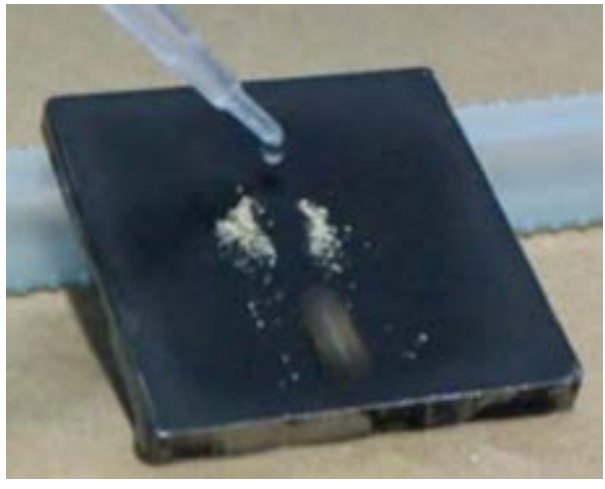

Fig. 3 Inspection on the self-cleaning property of the graphene superhydrophobic surface

As for the practical application in the outdoor environment, the superhydrophobic surface shall go through adverse external environment. This paper carries out the experiments of water and sand induction to the graphene superhydrophobic coating in order to study the mechanical resistance of the graphene superhydrophobic coating.. The superhydrophobic coating shall be placed with the angle of inclination in $45^{\circ}$, and the water droplet (10 microliters) shall impact the superhydrophobic surface from the height of $10 \mathrm{~cm}$. The graphene composite coating can keep its superhydrophobic property after the impacting of 18,000 water droplets. Besides, the superhydrophobic surface will be impacted by the dust grains easily in the external environment. In order to simulate the above-mentioned situation, this research places the sand with the diameter of $150 \mu \mathrm{m} \times 100 \mu \mathrm{m}$ at the height of $30 \mathrm{~cm}$ to impact the superhydrophobic surface. The graphene composite coating can keep its superhydrophobic property even if the weight of the sand reaches $50 \mathrm{~g}$.

\section{Conclusion}

In conclusion, the superhydrophobic coating, which is prepared by stripping the mixture of graphene and PDMS through electrochemical stripping, is of obvious self-cleaning property, as well as great tolerance to water and sand induction. The advantage of the research method used in this paper is simple process, because it's unnecessary to use the complicated equipment and expensive reagent or that regulated strictly for the preparation of graphene superhydrophobic coating. Therefore, a series of serious problems caused by ice coating can be solved by covering the graphene superhydrophobic coating on the insulator after the achievement of its controllable preparation. 


\section{References}

[1] Wen Xishan, Jiang Rikun, Jiang Xingliang, Bi Maoqiang, Shen Meng, The influence of the ice coating degree on the DC flashover performance of the ice-coated porcelain and glass insulator [J], High Voltage Engineering, 2010, (03):565-571.

[2] Jian Xin, Analysis on the anti-icing application of the superhydrophobic materials to the electrical power system [J], Science \& Technology Information, 2016, (22):31+33.

[3] H. Li, S. Yu, X. Han, Y. Zhao, A stable hierarchical superhydrophobic coating on pipeline steel surface with self-cleaning, anticorrosion, and anti-scaling properties, Colloids Surf. A 503 (2016) 43-52.

[4] P. Wang, M. Chen, H. Han, X. Fan, Q. Liu, J. Wang, Transparent and abrasion-resistant superhydrophobic coating with robust self-cleaning function in either air or oil, J. Mater. Chem. A 4 (2016) 7869-7874.

[5] Z. Zhang, B. Ge, X. Men, Y. Li, Mechanically durable, superhydrophobic coatings prepared by dual-layer method for anti-corrosion and self-cleaning, Colloids Surf. A 490 (2016) 182-188.

[6] X. Zhang, J. Liang, B. Liu, Z. Peng, Preparation of superhydrophobic zinc coating for corrosion protection, Colloids Surf. A 454 (2014) 113-118.

[7] A. Ganne, V. O. Lebed, A. I. Gavrilov, Combined wet chemical etching and anodic oxidation for obtaining the superhydrophobic meshes with anti-icing performance, Colloids Surf. A 499 (2016) 150-155.

[8] C. Liu, J. Yang, Y. Tang, L. Yin, H. Tang, C. Li, Versatile fabrication of the magnetic polymer-based graphene foam and applications for oil-water separation, Colloids Surf. A, 468 (2015) 10-16.

[9] K. Seo, M. Kim, S. Seok, D. H. Kim, Transparent superhydrophobic surface by silicone oil combustion, Colloids Surf. A 492 (2016) 110-118.

[10] Y. Zhao, Z. Xu, X. Wang, T. Lin, Superhydrophobic and UV-blocking cotton fabrics prepared by layer-by-layer assembly of organic UV absorber intercalated layered double hydroxides, Appl. Surf. Sci. 286 (2013) 364-370.

[11] K. Chang, H. Lu, C. Peng, M. Lai, S. Hsu, M. Hsu, Y. Tsai, C. Chang, W. Hung, Y. Wei, J. Yeh, Nanocasting technique to prepare lotus-leaf-like superhydrophobic elecroactive polyimide as advanced anticorrosive coatings, ACS Appl. Mater. Interfaces 5 (2013) 1460-1467.

[12] H. K. Park, S. W. Yoon, Y. R. Do, Superhydrophobicity of 2D SiO2 hierarchical micro/nanorod structures fabricated using a two-step micro/nanosphere lithography, J. Mater. Chem. 22 (2012) 14035-14041.

[13] S. S. Latthe, C. Terashima, K. Nakata, M. Sakai, A. Fujishima, Development of sol-gel processed semi-transparent and self-cleaning superhydrophobic coatings, J. Mater. Chem. A 2 (2014) 5548-5553.

[14] X. Deng, L. Mammen, H. Butt, D. Vollmer, Candle soot as a template for a transparent robust superamphiphobic coating, Science 335 (2012) 67-70.

[15] Y. Li, X. Men, X. Zhu, B. Ge, F. Chu, Z. Zhang, One-step spraying to fabricate nonfluorinated superhydrophobic coatings with high transparency, J. Mater. Sci. 51 (2016) 24112419.

[16] A. K. Geim, K. S. Novoselov, The rise of graphene, Nature Mater. 6 (2007) 183-191.

[17] Y. J. Shin, Y. Y. Wang, H. Huang, G. Kalon, A. T. S. Wee, Z. X. Shen, C. S. Bhatia, H. 
Yang, Surface-energy engineering of graphene, Langmuir 26 (2010) 3798-3802.

[18] J. Rafiee, M. A. Rafiee, Z. Z. Yu, N. Koratkar, Superhydrophobic to superhydrophilic wetting control in graphene films, Adv. Mater. 22 (2010) 2151-2154.

[19] J. Dong, Z. Yao, T. Yang, L. Jiang, C. Shen, Control of superhydrophilic and superhydrophobic graphene interface, Sci. Rep. 3 (2013) 1733.

[20] D. D. Nguyen, N. H. Tai, S. B. Leea, W. S. Kuob, Superhydrophobic and superoleophilic properties of graphene-based sponges fabricated using a facile dip coating method, Energy Environ. Sci. 5 (2012) 7908-7912.

[21] J. Wang, R. Shao, Y. Zhang, L. Guo, H. Jiang, D. Lu, H. Sun, Biomimetic graphene surfaces with superhydrophobicity and iridescence, Chem. -Asian J. 7 (2012) 301 -304.

[22] B. G. Choi, H. S. Park, Superhydrophobic graphene/nafion nanohybrid films with hierarchical roughness, J. Phys. Chem. C 116 (2012) 3207-3211.

[23] A. Asthana, T. Maitra, R. B chel, M. K. Tiwari, D. Poulikakos, Multifunctional superhydrophobic polymer/carbon nanocomposites: graphene, carbon nanotubes, or carbon black? ACS Appl. Mater. Interfaces 6 (2014) 8859-8867.

[24] N. Liu, F. Luo, H. Wu, Y. Liu, C. Zhang, J. Chen, One-Step ionic-liquid-assisted electrochemical synthesis of ionic-liquid-functionalized graphene sheets directly from graphite, Adv. Funct. Mater. 18 (2008) 1518-1525.

[25] K. Parvez, R. Li, S. R. Puniredd, Y. Hernandez, F. Hinkel, S. Wang, X. Feng, K. Müllen, Electrochemically exfoliated graphene as solution-processable, highly conductive electrodes for organic electronics, ACS Nano 7 (2013) 3598-3606.

[26] D. Wei, L. Grande, V. Chundi, R. White, C. Bower, P. Andrew, T. Ryhänen, Graphene from electrochemical exfoliation and its direct applications in enhanced energy storage devices, Chem. Commun. 48 (2012) 1239-1241.

[27] K. Chen, D. Xue, Preparation of colloidal graphene in quantity by electrochemical exfoliation, J. Colloid Interf. Sci. 436 (2014) 41 -46.

[28] K. Parvez, Z. Wu, R. Li, X. Liu, R. Graf, X. Feng, K. Müllen, Exfoliation of graphite into graphene in aqueous solutions of inorganic salts, J. Am. Chem. Soc. 136 (2014) 6083-6091.

[29] L. Buglione, E. L. K. Chng, A. Ambrosi, Z. Sofer, M. Pumera, Graphene materials preparation methods have dramatic influence upon their capacitance, Electrochem. Commun. 14 (2012) 5-8.

[30] J. Li, Z. Jing, Y. Yang, F. Zha, L. Yan, Z. Lei, Reversible low adhesive to high adhesive superhydrophobicity transition on ZnO nanoparticle surfaces, Appl. Surf. Sci. 289 (2014) 1 -5.

[31] R. E. Johnson, R. H. Dettre, Contact angle hysteresis. III. study of an idealized heterogeneous surface, J. Phys. Chem. 68 (1964) 1744-1750. 